\title{
BMJ Open Primary care micro-teams: a protocol for an international systematic review to describe and examine the opportunities and challenges of implementation for patients and healthcare professionals
}

\author{
Charles Richard Harvey Coombs (D) , ,2 Tanya Cohen, ${ }^{3}$ Claire Duddy (D) , \\ Kamal Ram Mahtani, ${ }^{4}$ Nia Roberts, ${ }^{4}$ Aman Saini, ${ }^{2}$ Alexander Staddon Foster, ${ }^{2}$ \\ Sophie Park ${ }^{1}$
}

To cite: Coombs CRH, Cohen T, Duddy C, et al. Primary care micro-teams: a protocol for an international systematic review to describe and examine the opportunities and challenges of implementation for patients and healthcare professionals. BMJ Open 2022;12:e052651. doi:10.1136/ bmjopen-2021-052651

- Prepublication history for this paper is available online To view these files, please visit the journal online (http://dx.doi. org/10.1136/bmjopen-2021 052651).

Received 21 April 2021 Accepted 28 January 2022

Check for updates

(c) Author(s) (or their employer(s)) 2022. Re-use permitted under CC BY-NC. No commercial re-use. See rights and permissions. Published by BMJ.

${ }^{1}$ Department of Primary Care and Population Health, UCL,

London, UK

${ }^{2}$ UCL Medical School, London, UK

${ }^{3}$ Patient and Public Contributor, London, UK

${ }^{4}$ Nuffield Department of Primary Care Health Sciences, University of Oxford, Oxford, UK

Correspondence to

Charles Richard Harvey Coombs; zchacoo@ucl.ac.uk

\section{ABSTRACT}

Introduction There has been a recent trend towards creating larger primary care practices with the assumption that interdisciplinary teams can deliver improved and more cost-effective services to patients with better accessibility. Micro-teams have been proposed to mitigate some of the potential challenges with practice expansion, including continuity of care. We aim to review the available literature to improve understanding of how micro-teams are described and the opportunities which primary care micro-teams can provide for practice staff and patients and limitations to their introduction and implementation. Our review asks: how is micro-team implementation described? What are the experiences of healthcare professionals and patients concerning micro-teams in primary care? What are the reported implications of microteams for patient care?

Methods and analysis CINAHL, Cochrane Library, Embase, MEDLINE and Scopus will be searched for studies in English. Grey literature will be sourced from Google Scholar, government websites, CCG websites, general practice directives and strategies with advice from stakeholders. Included studies will give evidence regarding the implementation of micro-teams. Data will be synthesised using framework analysis. We will use iterative stakeholder and public and patient participation to embed the perspectives of those whom micro-teams could impact. Included studies will be quality assessed using the Mixed Methods Appraisal Tool. The quality assessment will not be used to exclude any evidence but rather to develop a narrative discussion evaluating included literature.

Ethics and dissemination Ethical approval will not be necessary for this systematic review as there will only be a secondary analysis of data already available in scientific databases and the grey literature. This protocol has been submitted for registration to be made available on a review database (PROSPER0). Findings will be disseminated widely through peer-reviewed publication and in various media, for example, conferences, congresses or symposia.

PROSPERO registration number CRD42021225367.
Strengths and limitations of this study

- This protocol reduces the possibility for study duplication and offers transparency to the methods and analyses which will be used to reduce potential biases and give the opportunity to peer review.

- This will be the first systematic review to explore micro-teams in primary care and address a gap in the current evidence base by providing a comprehensive assessment of their implementation.

- The search strategy is not limited to nationality or time frame.

- Literature that is not available in English or an English translation will not be included which may lead to a language bias.

- There is the potential low and inconsistent quality evidence to be used as the quality assessment will be used to generate discussion instead of excluding literature; to mitigate this risk, we will address the quality of included studies in the discussion.

\section{INTRODUCTION}

In recent years, there has been a trend towards larger primary care practices with the concept that interdisciplinary teams can deliver improved and more cost-effective services to patients with better accessibility. ${ }^{1-4}$ With this expansion of patient numbers registered to general practices, there is a real threat that the continuity of care traditionally experienced in primary care will be lost. ${ }^{56}$ Larger practices may offer increased access and availability of resources, and provide a wider range of services. ${ }^{78}$ The benefits of larger practice sizes are, however, ambiguous given the lack of evidence that clinical outcomes or the patient's experience will improve. ${ }^{69}$

Continuity of care has been well documented to reduce both mortality and 
morbidity in patients as well as a reduction in secondary care referrals. Lack of continuity poses the threat of worsened patient and clinical outcomes. ${ }^{10-16}$ Over time, continuity of care enables clinicians to have a greater appreciation of their patient's personal circumstances and social history. This gives a personal context for healthcare priorities-commonly stated as placing the patient at the centre of care. ${ }^{17}$

Despite the perceived benefits, continuity of care has been in decline. ${ }^{18}$ The introduction of micro-teams has been proposed to mitigate some of the challenges resulting from practice expansion, in order to maintain a high level of continuity in patient care. 'Micro-team' is a term being introduced into the UK to encourage the organisation of mini multidisciplinary teams which serve a particular patient group within the practice, that is, teams within the wider multidisciplinary team of a practice. ${ }^{1920}$ In conjunction with a named general practitioner (GP) or primary care clinician, patients are able to build long-term relationships with several members of a multidisciplinary team. In addition to the established roles in general practices such as nursing and pharmacy, the team can potentially include emerging roles such as physician associates, paramedics, occupational therapists, line workers, dietitians and health coaches. ${ }^{19-23}$

The novelty of micro-teams has meant there is flexibility in which roles are incorporated into the team and there has been a range of roles reported. In the USA, micro-teams have been applied in the US Veterans Health Administration which employs the patient-centred medical home (PCMH) model of care. ${ }^{24}{ }^{25}$ The PCMH model can be implemented in a structure akin to microteams called teamlet. A teamlet is a small team, often organised as a clinician and healthcare assistant caring for a defined patient panel. ${ }^{26}$ The teamlet focuses on expanding the role of team-based structures to match patient needs. Within the UK, the Tower Hamlets GP Care Group micro-teams were piloted in their 2013/2014 strategy. Practices were free to define their own team model which could include a range of professionals with a variety of disciplines. ${ }^{23}$ This pilot was, however, deemed unsuccessful owing to three key issues: variation in understanding what is delivered in general practice; variation in leadership and decision-making across practices; and a lack of integrated data to analyse waste in the system. ${ }^{23} 27$ These two examples highlight the underlying international applicability of the micro-team concept.

Collaborative, interdisciplinary team-based care has been explored internationally, within the primary care setting. The Advancing Integrated Mental Health Solutions, Seattle, USA tackles common mental health conditions in a primary care setting through collaborative care. ${ }^{28}{ }^{29}$ In this model, a behavioural healthcare manager (BHCM) practices alongside the primary care team. The overall team works together to care for a population of patients with common mental health conditions such as depression and anxiety. The BHCM coordinates the overall effort of the team. They ensure effective communication among team members and the patient population. The model equips practices with the capacity to deliver both physical and mental healthcare.

The notion of collaboration and multidisciplinary team-based primary care is also advocated in Australia. The Royal Australian College of General Practitioners has published guidelines for optimising this in the community. Frameworks have been proposed to assist and support the implementation of team-based models of primary care within the Australian healthcare system. ${ }^{30}$ The importance of allied healthcare professionals working within primary care practices has been recognised. ${ }^{31-33}$ The evolution of roles such as community pharmacists offers a model of designated experts within the team, such as medication management. ${ }^{33}$ Integration of allied roles of primary care in Australia relies on government support through allocated remuneration. ${ }^{33}$

Guidelines released from the Royal College of General Practitioners highlight the importance of building a relationship of trust between each patient and their named key worker. They also raise the importance of responsibility and accountability for patient care. ${ }^{22}{ }^{34}$ The King's Fund has offered guidelines advocating the merit of micro-teams to develop ongoing patient-staff relationships, moving to a more proactive model of care. Additionally, routine work with the same team promotes trust among healthcare professionals. ${ }^{35} 36$

Our initial searching of electronic databases (International Prospective Register of Systematic Reviews (PROSPERO) and CDSR) in October 2020 confirmed the absence of any recent or ongoing relevant systematic reviews. This proposed systematic review aims to examine available literature to understand the opportunities and barriers to primary care micro-teams for practice staff and patients.

\section{Aim}

Review the available literature to improve understanding of how micro-teams are described and the opportunities which primary care micro-teams can provide for practice staff and patients and limitations to their introduction and implementation.

\section{Research question(s)}

1. How are micro-teams described?

2. What are the facilitators and barriers to micro-team implementation?

3. What are the experiences of healthcare professionals and patients concerning micro-teams in primary care?

\section{METHODS}

The synopsis for this systematic review protocol is registered in PROSPERO (registration number CRD42021225367).

We will complete a framework synthesis of the appropriate literature. We will initially scope the literature to familiarise and create a broad appreciation relating to how micro-teams have been used in primary care. Stakeholders and patient and public involvement (PPI) 


\begin{tabular}{|c|c|}
\hline Search & Term \\
\hline 1 & $\begin{array}{l}\text { (micro-team }{ }^{\star} \text { or microteam }{ }^{*} \text { or small team* or } \\
\text { teamlet*).ti,ab,kw. }\end{array}$ \\
\hline 2 & (micro-system or microsystem).ti,ab,kw. \\
\hline 3 & or/1-2 \\
\hline 4 & exp General Practice/ \\
\hline 5 & $\begin{array}{l}\text { general practitioners/ or physicians, family/ or } \\
\text { physicians, primary care/ }\end{array}$ \\
\hline 6 & Primary Health Care/ \\
\hline 7 & 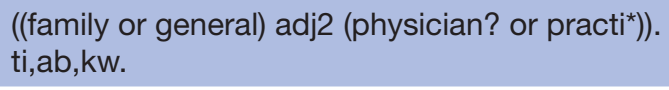 \\
\hline 8 & (primary adj2 (care or health»)).ti,ab,kw. \\
\hline 9 & or/4-8 \\
\hline 10 & 3 and 9 \\
\hline 11 & limit 10 to English language \\
\hline
\end{tabular}

contributors will be invited to contribute their perspectives at each stage of the review.

\section{Search strategy}

We will use a stepwise approach to searching: the initial step has been to scope the literature on MEDLINE to identify key terms and any suitable Medical Subject Headings (MeSH) terms to build a search strategy; the second step will be a comprehensive search of electronic databases including CINAHL, Cochrane Library, Embase, MEDLINE and Scopus with the predefined terms sourced from the initial scope. These are shown in table 1.

Each database will be searched in consultation with a librarian with expertise based on key search terms in the study selection criteria. All records of searches will be maintained for future reference to provide an audit trail to ensure transparency and perceived excellence. Grey literature will be sourced from Google Scholar, government websites, CCG websites, general practice directives and strategies with advice from stakeholders.

Grey literature is defined as unpublished studies, theses, presentations, website resources, government documents and other relevant documents which have not been published in journals and therefore do not appear in database searches. Grey literature will be included to minimise bias generated from only papers with positive findings being published. Often studies that have neutral, negative or limited outcomes are less likely to be published, in particular as this is an innovative area of evolving practice and policy change. ${ }^{37-39}$

Bibliographies of included literature will be searched to identify any relevant studies that were missed through the search strategy.

\section{Study selection criteria}

Inclusion criteria

- Written in English or existing translation available.
- Texts relevant to the design, development, evaluation or implementation of micro-teams in the primary care setting.

- Secondary data if available.

- Empirical studies using qualitative, quantitative or mixed methods approach; discursive or debate publications; or grey literature resources.

\section{Exclusion criteria}

- Studies with a focus on secondary care settings.

- Studies which use the term 'micro-system' to reference the contextual setting.

We will pilot these criteria with the research team to ensure they are clear and interpreted by each team member in as homogeneous a way as possible.

\section{Screening and data management}

Identified literature will be uploaded to the EPPIReviewer software. ${ }^{40}$ The software will be used to organise, collate and deduplicate search results. A Preferred Reporting Items for Systematic Review and Meta-Analyses flow diagram will be used to record the selection process, along with reasons for exclusion. ${ }^{41}$

EPPI-Reviewer software will be used to randomly allocate items to two independent reviewers. The literature will be disseminated so that CRHC reviews each citation while AS and ASF each double screen half of the total citations. This double screening process will be piloted on a sample of texts identified as core and peripheral texts, to ensure that each member of the team is clear about if or when a paper would be considered appropriate for inclusion.

All articles are to undergo a two-stage double screening process based on the inclusion criteria.

Stage 1: all citations will be screened based on their title and abstract. Reviewers will meet to discuss results. All disputed citations will be included for the full-text review.

Stage 2: the full text of each citation will be obtained. Each study will be read in full and assessed for inclusion.

Any 'unsure' texts will be discussed in the first instance between CRHC and SP, then with the whole research group. A record of discussions will be kept. Authors will be contacted for missing or incomplete information in order to determine inclusion. If no response from any viable methods of communication is recorded within 2 weeks, the article may be excluded based on missing information.

\section{Quality assessment}

Empirical studies relevant to this review might include a broad range of methods and methodological underpinnings. For example, ranging from quantitative comparisons of patient outcomes, through to sociological exploration of intrateam relationships. Quality assessment is therefore challenging and requires flexibility to adapt to the heterogeneity of research questions, methods and contexts. The Mixed Methods Appraisal Tool will be used to review the quality of the included studies. ${ }^{42}$ 
As the aim is to describe and synthesise the literature and not determine an effect size, studies will not be excluded based on the results of their quality assessment; the critical analysis will be used to develop a narrative discussion of the included studies.

\section{Framework synthesis}

A framework analysis approach will be used to extract and synthesise data. Framework synthesis is an adaptation of 'framework analysis' originally developed to analyse primary research data. ${ }^{43} 44$ Analysis will be deductive to explicitly address our research questions and inductive analysis to enable us to respond to the emergent themes identified within the data. The emergent element to thematic summaries will allow an initial conceptual framework to evolve during the synthesis as reviewers become more familiar with the literature and aware of key ideas and recurrent themes. This will allow for an 'emergent framework' to the modified conceptual framework. Micro-teams are an innovative area of evolving practice organisation and policy change, with no known previous systematic analysis according to our initial scoping of the literature and search of PROSPERO and CDSR, an iterative approach is therefore desirable.

Additionally, the development of higher-order themes can potentially confirm the initial framework or enhance the understanding of the initial framework-to go beyond what a simple descriptive thematic summary would produce.

The framework synthesis will be carried out in two broad stages:

\section{Stage 1: developing or selecting an initial framework}

\section{Familiarisation}

- Framework synthesis will start with familiarisation of the literature available.

- Notes taken from the familiarisation phase will highlight key issues, concepts and themes.

2. Developing an initial thematic framework

- An initial conceptual framework will guide the extraction and combination of data. The notes taken from the familiarisation phase will form the basis of a thematic framework that can be used to filter and classify the data. The intention is that the framework will evolve as the reviewers interact with the literature. The initial framework will be based on the initial scoping of the literature.

- The initial framework will be informed by: current policy; direct experience of the research team, including stakeholders and PPI; themes which become apparent from abstracts identified through the initial search strategy.

\section{Stage 2: recognising patterns through aggregation}

\section{Indexing}

- Revealing data available will be deductive and inductive, using an iterative process of aggregating and reconfiguring the data.
- Themes will be expanded as their significance becomes more apparent.

- To analyse the findings of the selected qualitative studies, a 'thematic synthesis analysis' will be performed, using EPPI-Reviewer V.4 software (UCL Institute of Education, University of London, UK).

2. Charting

- Each element of the framework will be tackled in turn, tabulating the data under key themes to present distilled summaries. The data aggregated according to themes or dimensions within the final configuration of the emergent framework.

3. Mapping

- Drawing together what can be learnt from the tables and summaries. Creating typologies, mapping the multidimensional nature and diversity of phenomena, finding associations between themes and providing explanations for the findings across the included literature.

There is a risk with qualitative systematic reviews that the essence of the original literature theory is lost. The interpretations are secondary and from others' interpretations. This means that first the data processing must be structured in a way that the review's findings are firmly rooted in the original data to ensure it echoes the original participants' views. Second, the process of interpretation and synthesis is reflexive and explicit to enable readers to understand (and potentially replicate) how the review was done. A record will therefore be kept for how decisions we made and evolved to support analysis.

Data analysis will primarily be undertaken by CRHC. Iterative 'data clinics' will be conducted with the research team and periodical discussion of emergent findings with stakeholder and PPI groups. This will aim to maximise the relevance and utility of findings for practice organisation and patient care.

\section{PPI and stakeholder involvement}

Stakeholders in systematic reviews have been described in a variety of ways. ${ }^{45}$ Stakeholders are defined as those who have a 'self-interest' in the piece of work. The results of the research may impact on their current work practices. Due to the flexibility of roles which can be incorporated into micro-teams, stakeholders will include all practice staff as well as patients. Practice staff is defined as any individual who professionally works in a primary care practice.

One coauthor (TC) is a PPI representative and has been involved from the inception and development of the review protocol. She will be closely involved in the iterative analysis of the review. She has helped shape the research aim and questions to aid the identification and prioritisation of research areas to ensure research priorities are aligned with those of patients.

There will be periodical dialogue with a stakeholder advisory group. This comprises a diverse range of healthcare professionals and practice staff whose work has involved or might potentially be relevant to primary 
care micro-teams as well as patients. This project will be presented to the PPI representative and the stakeholder advisory group at the protocol and stage of emergent findings. We will invite comments on the protocol, emergent data, potential audiences and potential key messages for recommendations from this study.

Sampling strategy criteria for stakeholders include multidisciplinary practice roles and primary care network (PCN) managerial roles; a range of practice size (staff and patient numbers) and patient history (population demographic, condition). We have agreement from a number of primary healthcare staff including GPs, PCN committee members, practice managers and other healthcare professionals. These individuals have been sampled from a range of practice sizes and according to differing longevities of practice arrangements, that is, mergers.

\section{Ethics and dissemination}

Ethical approval will not be necessary for this systematic review as there will only be a secondary analysis of data already available in scientific databases and the grey literature. As this will be a systematic review of existing without individual primary data, there will be no need for ethical approval.

This protocol is available on a review database (PROSPERO). This is to maximise transparency of the review methodology and allow for peer review and feedback before the study is completed. Having a published protocol enhances trustworthiness in the subsequent review findings and recommendations.

Following stakeholder involvement at protocol and analysis stages, we will discuss dissemination possibilities and plan to maximise the reach and relevance of this review to inform future practice and patient care. Dissemination will include the stakeholder and PPI advisory group, various media such as conferences (eg, Madingley SAPC), seminars (PCPH departmental), congresses or symposia, and social media (eg, Twitter chat, SPCR blog).

\section{Output and impact}

This review is designed to synthesise the available literature to understand the effectiveness which primary care micro-teams have for practice staff and patients. This study will incorporate the views and priorities of both healthcare workers and patients as stakeholders into the introduction of primary care micro-teams.

How primary care is organised has the potential to impact on the nature and quality of patient care. ${ }^{46}$ This review will bring together a range of literature about how, in what manner and why micro-teams have been considered and used in the primary care setting. Through our analysis, we will identify key findings from these papers which will help to inform recommendations for future practices about the opportunities and challenges which micro-team adoption might present to healthcare professionals and patients.
We used the Preferred Reporting Items for Systematic Review and Meta-Analysis Protocols checklist when writing our protocol. ${ }^{41}$

Contributors $\mathrm{CRHC}$ and SP conceived the systematic review. All authors contributed to establishing research questions, aims and methods of the proposed review. Initial literature searches were performed by CRHC, CD and NR to establish suitable search terms. TC was strongly involved in the participation of stakeholders and patient and public contributors. CRHC wrote the initial draft of the protocol which TC, CD, ASF, KRM, NR, AS and SP revised. Subsequent drafts were commented on by all members of the review team. All authors read and approved the final manuscript.

Funding The authors have not declared a specific grant for this research from any funding agency in the public, commercial or not-for-profit sectors.

\section{Competing interests None declared.}

Patient and public involvement Patients and/or the public were involved in the design, or conduct, or reporting, or dissemination plans of this research. Refer to the Methods section for further details.

Patient consent for publication Not required.

Provenance and peer review Not commissioned; externally peer reviewed.

Open access This is an open access article distributed in accordance with the Creative Commons Attribution Non Commercial (CC BY-NC 4.0) license, which permits others to distribute, remix, adapt, build upon this work non-commercially, and license their derivative works on different terms, provided the original work is properly cited, appropriate credit is given, any changes made indicated, and the use is non-commercial. See: http://creativecommons.org/licenses/by-nc/4.0/.

\section{ORCID iDs}

Charles Richard Harvey Coombs http://orcid.org/0000-0002-2023-6462

Claire Duddy http://orcid.org/0000-0002-7083-6589

\section{REFERENCES}

1 Morciano M, Checkland K, Hammond J, et al. Variability in size and characteristics of primary care networks in England: observational study. Br J Gen Pract 2020;70:e899-905.

2 Devlin RA, Hogg W, Zhong J, et al. Practice size, financial sharing and quality of care. BMC Health Serv Res 2013;13:446.

3 Goodwin N, Dixon A, Poole T. Improving the quality of care in general practice: report of an independent inquiry commissioned by the King's Fund. London: King's Fund, 2011.

4 Ireland, ed. Primary care: a new direction: quality and fairness - a health system for you. Dublin: Stationery Office, 2001.

5 CWL N, KP N. Does practice size matter? Review of effects on quality of care in primary care. Br J Gen Pract 2013;63:e604-10.

6 Pineault R, Provost S, Borgès Da Silva R, et al. Why is bigger not always better in primary health care practices? the role of mediating organizational factors. Inquiry 2016;53. doi:10.1177/0046958015626842. [Epub ahead of print: 31012016 ].

7 Palmer W, Hemmings N, Rosen R. Improving access and continuity in general practice. Nuffield Trust 2018;28.

8 Schneebacher J, Ardanaz-Badia A, Hilton Z. Management practices in Great Britain - Office for National Statistics, 2021. Available: https://www.ons.gov.uk/economy/economicoutputandproductivity/ productivitymeasures/articles/managementpracticesingreatbritain/ 2016to2020 [Accessed 3 Nov 2021].

9 Pettigrew LM, Kumpunen S, Rosen R, et al. Lessons for 'largescale' general practice provider organisations in England from other inter-organisational healthcare collaborations. Health Policy 2019;123:51-61.

10 Baker R, Freeman GK, Haggerty JL, et al. Primary medical care continuity and patient mortality: a systematic review. Br J Gen Pract 2020;70:e600-11.

11 Hofer A, McDonald M. Continuity of care: why it matters and what we can do. Aust J Prim Health 2019;25:214.

12 Jeffers $\mathrm{H}$, Baker M. Continuity of care: still important in modern-day general practice. Br J Gen Pract 2016;66:396-7.

13 Pereira Gray DJ, Sidaway-Lee K, White E, et al. Continuity of care with doctors - a matter of life and death? A systematic review of continuity of care and mortality. BMJ Open 2018;8:e021161.

14 Mahase E. Mortality rates are lower with higher continuity of care, review finds. BMJ 2020:m3184. 
15 Hansen AH, Halvorsen PA, Aaraas IJ, et al. Continuity of GP care is related to reduced specialist healthcare use: a cross-sectional survey. Br J Gen Pract 2013;63:e482-9.

16 Gray DP, Evans P, Sweeney K, et al. Towards a theory of continuity of care. J R Soc Med 2003;96:160-6.

17 Paddison C. How can we get better at providing patient centred care: does continuity matter? BMJ 2015;350:h1127.

18 Levene LS, Baker R, Walker N, et al. Predicting declines in perceived relationship continuity using practice deprivation scores: a longitudinal study in primary care. Br J Gen Pract 2018;68:e420-6.

19 Baird B. How has general practice responded to the Covid-19 (coronavirus) outbreak? 2020. The King's Fund. Available: https:// www.kingsfund.org.uk/blog/2020/04/covid-19-general-practice [Accessed 27 Apr 2021].

20 Smith A. 15-minute minimum consultations, continuity of care through "micro-teams", and an end to isolated working: this is the future of general practice, 2019. Available: https://www.rcgp.org. uk/about-us/news/2019/may/15-minute-minimum-consultationscontinuity-of-care.aspx [Accessed 17 Nov 2020].

21 Bienkowska-Gibbs T, King S, Saunders CL. New organisational models of primary care to meet the future needs of the NHS: a brief overview of recent reports. RAND Europe 2015;59.

22 Fit for the future. A vision for general practice.

23 Risi L, Bhatti N, Cockman P, et al. Micro-teams for better continuity in Tower Hamlets: we have a problem but we're working on a promising solution! Br J Gen Pract 2015;65:536.

24 Rosland A-M, Nelson K, Sun $\mathrm{H}$, et al. The patient-centered medical home in the Veterans health administration. Am J Manag Care 2013;19:e263-72.

25 Nelson KM, Helfrich $\mathrm{C}$, Sun $\mathrm{H}$, et al. Implementation of the patientcentered medical home in the Veterans health administration: associations with patient satisfaction, quality of care, staff burnout, and hospital and emergency department use. JAMA Intern Med 2014;174:1350-8.

26 Bodenheimer T, Laing BY. The teamlet model of primary care. Ann Fam Med 2007;5:457-61.

27 Risi L, Kullar B. Fresh doctors and cost effective care in tower Hamlets. handy approach to care, 2018. Available: https:// handyapproachtocare.com/2018/07/06/fresh-doctors-and-costeffective-care-in-tower-hamlets/ [Accessed 11 Jan 2021]

28 Unützer J, Katon W, Callahan CM, et al. Collaborative care management of late-life depression in the primary care setting: a randomized controlled trial. JAMA 2002;288:2836-45.

29 Archer J, Bower P, Gilbody S, et al. Collaborative care for depression and anxiety problems. Cochrane Database Syst Rev 2012;10:CD006525.

30 Naccarella L, Greenstock LN, Brooks PM. A framework to support team-based models of primary care within the Australian health care system. Med J Aust 2013;199:199.
31 Cheong LH, Armour CL, Bosnic-Anticevich SZ. Multidisciplinary collaboration in primary care: through the eyes of patients. Aust $J$ Prim Health 2013;19:190-7.

32 Reeves S, Pelone F, Harrison R, et al. Interprofessional collaboration to improve professional practice and healthcare outcomes. Cochrane Database Syst Rev 2017;6:CD000072.

33 Dineen-Griffin S, Benrimoj SI, Garcia-Cardenas V. Primary health care policy and vision for community pharmacy and pharmacists in Australia. Pharm Pract 2020;18:18.

34 Mulholland M. Creating effective GP practices in future. Available: https://www.rcgp.org.uk/clinical-and-research/about/clinicalnews/2018/august/creating-effective-gp-practices-in-future.aspx [Accessed 10 Jan 2021].

35 Baird B, Charles A, Honeyman M. Understanding pressures in general practice. 100. The King's Fund, 2016.

36 Baird B, Boyle T, Chauhan K. How to build effective teams in general practice. The King's Fund, 2020. Available: https://www.kingsfund. org.uk/publications/effective-teams-general-practice [Accessed 18 Nov 2020].

37 Paez A. Grey literature: an important resource in systematic reviews. J Evid Based Med 2017.

38 Higgins J, Thomas J, Chandler J. Cochrane Handbook for systematic reviews of interventions. /handbook/current.

39 Mahood Q, Van Eerd D, Irvin E. Searching for grey literature for systematic reviews: challenges and benefits. Res Synth Methods 2014;5:221-34.

40 Thomas J, Brunton J, Graziosi G. EPPI-Reviewer 4.0: software for research synthesis. EPPI-Centre software. London: Social Science Research Unit, Institute of Education, University of London, 2010.

41 Moher D, Liberati A, Tetzlaff J, et al. Preferred reporting items for systematic reviews and meta-analyses: the PRISMA statement. PLoS Med 2009;6:e1000097.

42 Hong QN, Fàbregues Feijóo S, Bartlett G. The mixed methods appraisal tool (MMAT) version 2018 for information professionals and researchers. Available: http://openaccess.uoc.edu/webapps/o2/ handle/10609/93312 [Accessed 31 May 2021].

43 Ritchie J, Spencer L. Qualitative data analysis for applied policy researchqualitative data, 1994.

44 Gough D, Thomas J, Oliver S. Clarifying differences between review designs and methods. Syst Rev 2012;1:28.

45 Abrams R, Park S, Wong G, et al. Lost in reviews: looking for the involvement of stakeholders, patients, public and other nonresearcher contributors in realist reviews. Res Synth Methods 2021;12:239-47.

46 Park S, Abrams R, Wong G, et al. Reorganisation of general practice: be careful what you wish for. $\mathrm{Br} J$ Gen Pract 2019;69:517-8 